\title{
Response Process Validation Protocol Using Neurophenomenological Gamma Asymmetry
}

\author{
Ron Bonnstetter*, Eric Gehrig, and Dustin Hebets \\ Target Training International Success Insights, Scottsdale, Arizona, USA
}

\begin{abstract}
In the process of designing a continuous improvement process for a set of personal attribute self-reporting assessments, an opportunity was seen to combine assessment analytics with brain activity to capture decisionmaking pathways while responding to assessment items. This pilot triangulation process is designed to address response process validation, as described by the American Psychological Association, to begin to better understand whether the responder is following the intended purpose of the assessment. Method: The protocol involves collecting electroencephalographic (EEG) data, using standardized low-resolution brain electromagnetic tomography (sLORETA) to analyze and view voxel images of real-time brain activity collected while a participant responds to assessment items. This analysis examines gamma asymmetry in the frontal lobes, as well as opens the door to further wave comparisons in the future. Conclusions: The protocols used to expose the mindset of assessment responders will be shared, as well as pilot insights gained as a result of this imaging process. By collecting images from the moment of stimulus exposure to the moment that the respondent selects a Likert scale answer, insights are gathered that include: how final answers compare to brain processing data, brain decisionmaking pathways when exposed to reverse or double negative assessment items, exposure of brain processing when faced with socially loaded statements, resulting brain processing of neutrally scored stimulus, and insights gained when all of this data is crosswalked against quantitative item analysis of population data such as interitem correlations and item factor loading based on exploratory factor analysis.
\end{abstract}

Keywords: gamma asymmetry; response process validity; industrial/organizational psychology; organizational neuroscience; sLORETA; approach-avoidance; data triangulation

Citation: Bonnstetter, R., Gehrig, E., \& Hebets, D. (2018). Response process validation protocol using neurophenomenological gamma asymmetry. NeuroRegulation, 5(3), 93-102. http://dx.doi.org/10.15540/nr.5.3.93

*Address correspondence to: Ron Bonnstetter, PhD, TTI SI, 17785 Edited by:

N. Pacesetter Way, Scottsdale, AZ 85255, USA. Email: Rex L. Cannon, PhD, Knoxville Neurofeedback Group, Knoxville, ron@ttiltd.com

Tennessee, USA

Copyright: () 2018. Bonnstetter et al. This is an Open Access article distributed under the terms of the Creative Commons Attribution License (CC-BY).

\section{Reviewed by:}

Rex L. Cannon, PhD, Knoxville Neurofeedback Group, Knoxville, Tennessee, USA

Randall Lyle, PhD, Mount Mercy University, Cedar Rapids, lowa, USA

The American Psychological Association (APA) Handbook of Testing and Assessment in Psychology states that assessment response processes require the collection of evidence demonstrating that the test taker is cognitively processing and properly interpreting the intended purpose of test items (Sireci \& Sukin, 2013). This form of validation evidence is used to demonstrate that the assessment directs participants to engage in specific behaviors deemed necessary to complete the designed purpose of the assessment items.

For instance, if an assessment is designed to measure self-reported behaviors, instructions should clarify the mindset required to fulfill that task. Here is an example of revised introductory instructions from our Style Insights behavioral assessment (TTI Success Insights, 2018).

The Style Insights Instrument is designed to identify observable human behavior. This assessment consists of 24 sets of descriptors. The descriptors are designed to reflect a range that describes you. The order that you use to select items is up to you. Please do not overanalyze, use your first impression. There are no right or wrong answers. Please complete the assessment in one sitting. While there is no 
time limit, the typical individual takes approximately 10 minutes.

While the instructions may lay the groundwork for directing the mindset of a respondent and are a crucial component, the APA handbook goes on to point out just how difficult a task this request is to accomplish. They state that:

Gathering validity evidence based on response process is perhaps the most difficult validity evidence to gather because it involves demonstrating that examinees are invoking the hypothesized constructs the test is designed to measure in responding to test items. As the Standards describe [2], "Theoretical and empirical analyses of the response processes of test takers can provide evidence concerning the fit between the construct and the detailed nature of performance or response actually engaged in by examinees." Gathering this type of evidence is difficult because one cannot directly observe the cognitive processes going on within people's heads as they respond to test items. Although some studies have used MRI to see which regions of the brain are activated when responding to tasks, most studies of response processes use indirect means such as cognitive interviews, think-aloud protocols, focus groups, or analysis of answer patterns and item response time data and attempt to set the stage with introductory statements of purpose (Sireci \& Sukin, 2013, p. 76).

Until recently this validation requirement has been virtually impossible to accomplish neurologically. There have been magnetic resonance imaging (MRI) studies that show regions of the brain that activate when responding to various stimuli (Owen, Borowsky, \& Sarty, 2004), but MRI only shows resulting brain activity based on blood flow and is, therefore, unable to detect the moment by moment decision-making pathways leading to the respondent's cognitive processing. In addition, MRI is uncomfortable for many participants, can be emotionally upsetting due to prolonged confinement, is expensive to operate and virtually impossible to design protocols that allow a client to observe a stimulus and respond in real time.

Electroencephalography (EEG), on the other hand, measures voltage fluctuations within specific neural networks or regions, thus recording the brain's spontaneous electrical activity and thereby exposing real-time brain decision-making pathways. In June 2015, Target Training International (in conjunction with Thomas Collura, $\mathrm{PhD}$ ) was issued a patent on their Validation Process for Ipsative Assessments (Bonnstetter, Bonnstetter, Hebets, \& Collura, 2015). The patent abstract reads:

This invention is a validation process for ipsative assessments. Respondents are connected to an electroencephalograph (EEG) and some or all of the ipsative assessment questions are asked again while connected to the EEG. The EEG measuring frontal lobe responses in terms of gamma waves is compared with the assessment questions. Positive responses provide one frontal lobe response in terms of gamma waves, negative or false answers provide a different gamma response and neutral questions provide a neutral gamma response. Reading the responses then tells whether the respondent initially responded with integrity (Bonnstetter, Bonnstetter, et al., 2015, p. 1).

Detailing the protocols used for our internal response validation is beyond the scope of this paper but can be assessed by reading (Bonnstetter, Bonnstetter, et al., 2015; Collura, Wigton, Zalaquett, Chatters-Smith, \& Bonnstetter, 2016; Collura, Zalaquett, Bonnstetter, 2014; Collura, Zalaquett, Bonnstetter, \& Chatters, 2014).

In general, the Gamma for Ipsative Validation using Electroencephalography (GIVE) process accesses asymmetric gamma wave bursts in the prefrontal cortex to validate the underlying subconscious decisions behind these self-reported responses, at the very moment of decision-making. As stated in the patent:

Until now no process has linked these specific types of self-reports to actual brain activity. The new process uses asymmetric wave analysis resulting from a stimulus to validate the underlying mental decisions behind these reported responses at the very moment of decision-making, thus exposing the true thoughts behind their responses and documenting potential abnormalities between their pre-assessments and their actual brain activity. This process provides evidence that an evoked emotionally laden response results in corresponding brain activity and documents both the intensity of human emotional response as well as the directionality of the response" (Bonnstetter, Bonnstetter, et. al., 2015, p. 7-8).

Figure 1 is an example of frontal lobe gamma asymmetry with approach, neutral, and avoidance 
responses. The orientation of the brain is facing forward such that the right hemisphere is on the left side of the image. Red colors indicate an increase in gamma activity, blue colors indicate a decrease in gamma activity, and green colors are indicative of little or no activation.

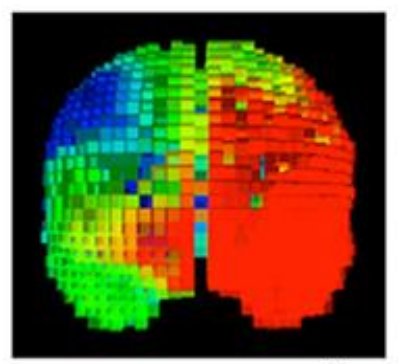

Approach

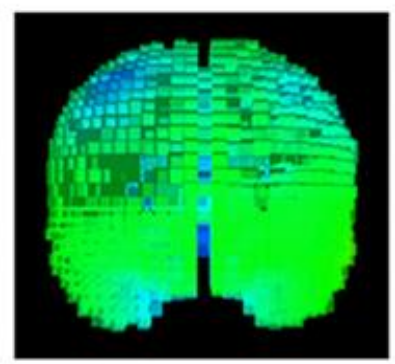

Neutral

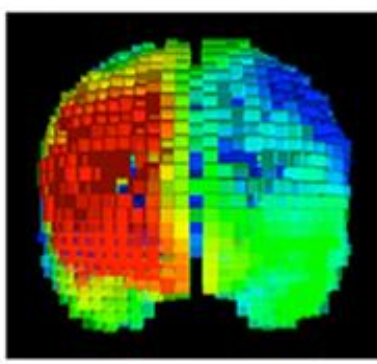

Avoidance

Figure 1. Frontal Lobe Gamma Asymmetry Summary.

At this point it is important to draw attention to the last patent sentence quoted above, "This process provides evidence that an evoked emotionally laden response results in corresponding brain activity and documents both the intensity of human emotional response as well as the directionality of the response" (Bonnstetter et al., 2015, p. 8).

Figure 2 depicts data from one of our behavioral assessment frames. In this example, the respondent first took our online assessment and, as shown, ordered the four behavioral choices from 1 to 4 , with 1 being the most like them and 4 being the least descriptive of their behavioral style. Within two weeks of the initial assessment, they were placed in our EEG lab and given the same task with their brain activity being collected in real time.

The frontal lobe images in Figure 2 show classic asymmetry acceptance and avoidance responses to the stimuli. Their number 1 choice (the item that was "most like them") has a left dominant gamma wave activity that depicts acceptance of the concept while their number 4 choice clearly shows a rightside reaction, depicting avoidance. It is also interesting to note that 2 and 3 have very little gamma activity to the stimuli and a mixed asymmetry.

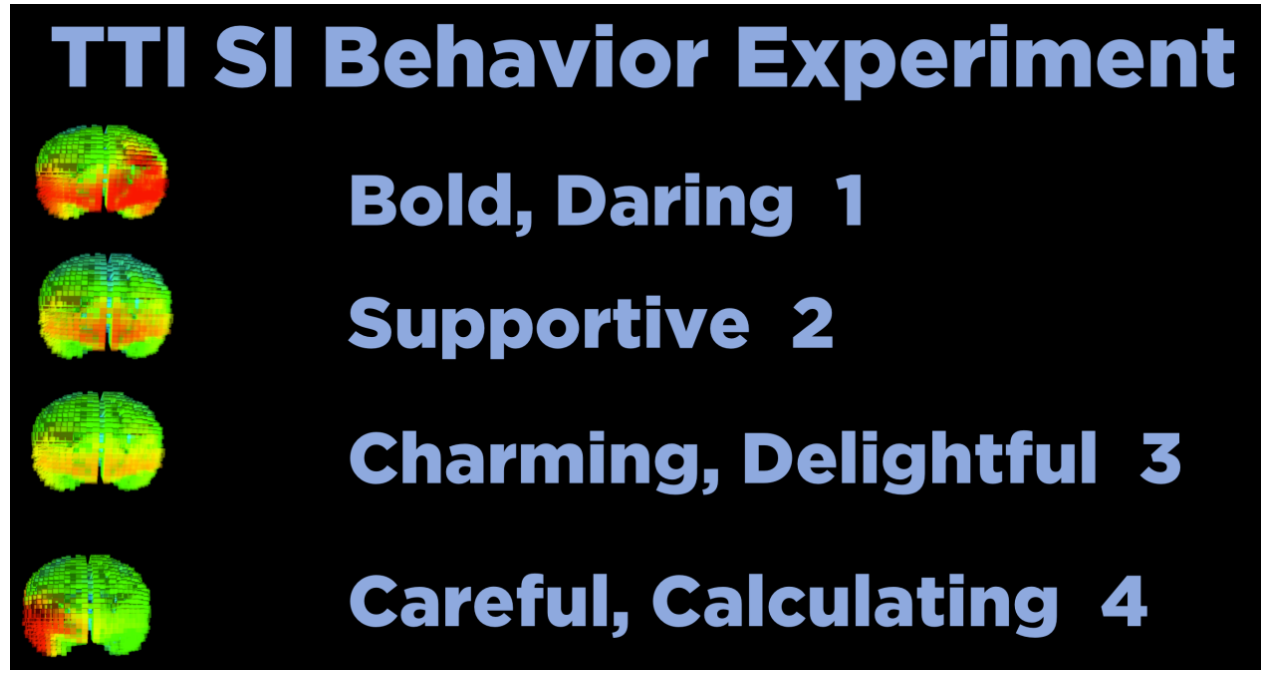

Figure 2. Sample Survey Responses and Corresponding Brain Images. 
The significance of this data lies in the fact that this process lies at the heart of our ongoing efforts for continuous improvement by learning about our assessments in ways that open new insights daily. For example, our internal study of our behavior assessment shows that we can match the most liked respondent's choice to brain activity $86 \%$ of the time and we are able to match the least liked items $97 \%$ of the time. This gives us insights into how our brain is much clearer regarding our dislikes than our likes, which is valuable information as we work toward continuous improvement and also provides direct insights into the respondents' mental processing.

While the application on ipsative assessments in our initial work with behaviors was only interested in identifying decision-making directionality (see Figure 2), Likert-scaled assessments allow the ability to expose not only acceptance or rejection but also provide insights into the degree or intensity of the decision process.

As can be seen in Figure 3, reproduced from Bonnstetter, Hebets, and Wigton (2015), not only do we see right and left prefrontal cortex asymmetry and the visual expression of intensity, we find a list of qEEG (quantitative electroencephalograph) data that provides a measure of the emotional response intensity for each soft skill stimuli. These quantitative values are the average of the ROI (region of Interest) voxels for the right and left frontal lobes. A measure of acceptance versus avoidance is calculated in the form of a numeric difference, termed the Approach-Avoidance-Differential (AAD), indicating the relative amount of energy in the right frontal lobe ROI, compared to the left. The AAD calculation is the average of the right hemisphere ROI, voxels minus the average of the left hemisphere ROI voxels $(1,088$ voxels per hemisphere). A negative value indicates greater left hemisphere activation and implies approach (i.e., a sense of accepting thoughts, feelings, and behavior) towards the stimulus word; a positive value indicates greater right hemisphere activation and implies avoidance (i.e., a sense of aversion) against the stimulus word; and a value near zero implies a neutral response.

Figure 4 describes the process used to collect brain activity data from our TTI Success Insights Emotional Quotient. The 57 assessment items are presented one at a time, just as they are presented in the online assessment platform. Each assessment item is on the screen for $2.8 \mathrm{~s}$ to capture initial emotional reactions, followed by a second screen that reiterates the stimuli and provides a six-choice Likert scale that ranges from very inaccurate to very accurate. This second screen remains active until the respondent clicks on their answer at which time a blank screen appears for a random period of two to five seconds. After the rest period, a new item stimulus is introduced.

The EEG amplifier was the Discovery 24E (BrainMaster Technologies, Bedford, $\mathrm{OH}$ ) with a sampling rate of 1,024 samples per second (data rate to the computer of 256 samples per second), an $\mathrm{A} / \mathrm{D}$ conversion of 24-bit resolution, EEG bandwidth of $0.43-80 \mathrm{~Hz}$, and input impedance of $1,000 \mathrm{Gohm}$. EEG is acquired with the BrainAvatar software (BrainMaster Technologies, Bedford, $\mathrm{OH}$ ) with linked ears reference; electrode impedance is adjusted to be below $10 \mathrm{kohm}$.

During the stimuli presentation, two auxiliary channels of the amplifier were used to record event start and stop markers. These markers were generated using a predesigned random set of emotional intelligent questionnaire stimuli built into an E-Prime 2.0 software (Psychology Software Tools, Inc., Sharpsburg, PA). Prior to presenting the stimuli, 2 min of eyes-open and 2 min of eyes-closed EEG were collected for further analysis and to document baseline status. 
Frontal Gamma Asymmetry in Response to Soft Skills Stimuli in a Single Case (Participant \#1)

\begin{tabular}{|c|c|c|c|c|c|c|c|}
\hline $\begin{array}{l}\text { Rank } \\
\text { Order } \\
\end{array}$ & $\begin{array}{l}\text { TriMetrix }\left({ }^{\circledR}\right) \text { DNA } \\
\text { Soft Skill } \\
\end{array}$ & AAD & $\begin{array}{l}\text { Gamma } \\
\text { Image }\end{array}$ & $\begin{array}{l}\text { Rank } \\
\text { Order } \\
\end{array}$ & $\begin{array}{c}\text { TriMetrix@ DNA } \\
\text { Soft Skill } \\
\end{array}$ & AAD & $\begin{array}{l}\text { Gamma } \\
\text { Image }\end{array}$ \\
\hline 1 & Presenting & -0.91 & & 13 & Teamwork & 0.19 & \\
\hline 2 & Diplomacy & -0.899 & & 14 & Management & 0.31 & \\
\hline 3 & Customer Service & -0.86 & & 15 & Conflict Management & 0.27 & \\
\hline 4 & Self-Management & -0.40 & & 16 & $\begin{array}{l}\text { Analytical Problem } \\
\text { Solving }\end{array}$ & 0.11 & \\
\hline 5 & Interpersonal Skills & -0.37 & & 17 & Decision Making & 0.09 & \\
\hline 6 & $\begin{array}{l}\text { Employee } \\
\text { Development/ } \\
\text { Coaching }\end{array}$ & 0.21 & & 18 & $\begin{array}{l}\text { Creativity/ } \\
\text { Innovation }\end{array}$ & 0.10 & \\
\hline 7 & Continuous Learning & -0.39 & & 19 & $\begin{array}{c}\text { Personal } \\
\text { Effectiveness }\end{array}$ & 0.07 & \\
\hline 8 & Planning/ Organizing & 0.66 & & 20 & Futuristic Thinking & 0.19 & \\
\hline 9 & Persuasion & 0.58 & & 21 & Leadership & 0.21 & \\
\hline 10 & $\begin{array}{c}\text { Written } \\
\text { Communication }\end{array}$ & 0.47 & & 22 & Negotiation & 0.15 & \\
\hline 11 & Empathy & 0.32 & & 23 & Flexibility & 0.03 & \\
\hline 12 & Goal Orientation & 0.17 & & $\begin{array}{l}\text { Note. AA } \\
\text { correspor } \\
\text { being the } \\
\text { the image }\end{array}$ & $\begin{array}{l}\text { = Approach-Avoidanc } \\
\text { to brain activity, gar } \\
\text { ighest and dark blue th } \\
\text { s the participant's left h }\end{array}$ & $\begin{array}{l}\text { ifferen } \\
\text { a freq } \\
\text { west. } \\
\text { isphere }\end{array}$ & blors \\
\hline
\end{tabular}

Figure 3. Source: Bonnstetter, Hebets, \& Wigton, 2015. 
SLIDE 1

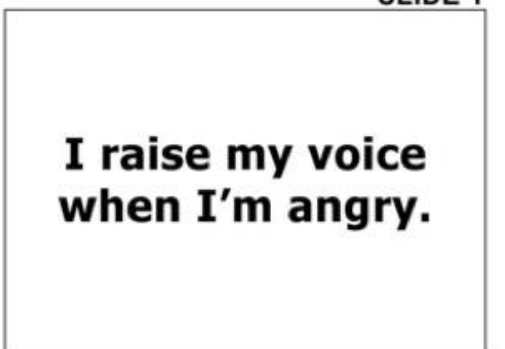

2.8 Secs

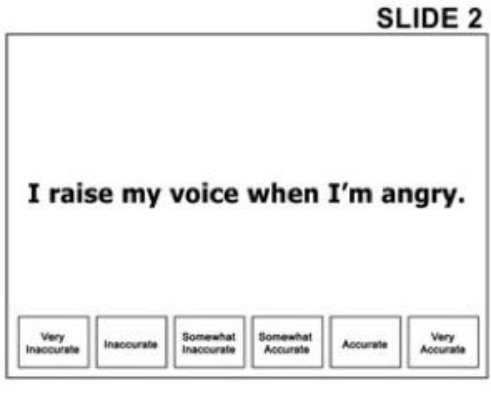

(Hold)

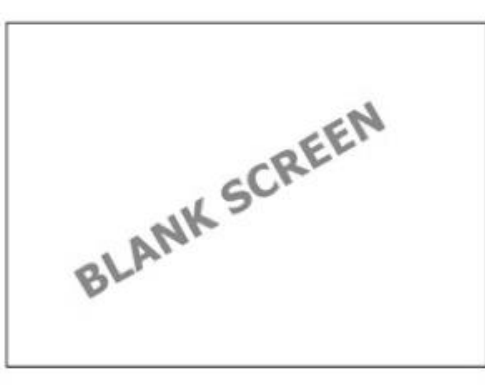

2 to 5 Secs

Figure 4. Sample Stimuli Screen Presentation.

Although complete descriptions of resulting insights are not the focus of this paper, the authors will discuss several examples depicting this imaging process and how it connects to response process validation.

During postprocessing analysis, averaged voxel values and SLORETA images are matched to the individual emotional quotient stimuli and compared to the final selected item answer. The ROI for analysis is identified as "frontal lobe," as predefined in the BrainAvatar imaging software (as designated by the Key Institute sLORETA model) and includes only the left and right frontal lobes for this first round of analysis. For purposes of remaining on task, the authors are only addressing the sLORETA imaging data at this time. However, additional insights are emerging as the authors cross-reference the images with the previously performed population statistical analysis including item analysis and factor loading.

Figures 5-7 provide several sample outputs from a pilot TTI Success Insights Emotional Quotient assessment, based on data from the participant's exposure to slide 1. A similar processing protocol is used for the second slide data that will not be presented in these examples, partly because we are seeing most, if not all, of the gamma processing during this first phase. The actual item statement being used as the sample stimuli is unimportant to understanding the process and to some extent represents proprietary information.

Because of varying baselines between individual participants and therefore creating difficulty with image comparisons, all images are from the same respondent. Note that BrainAvatar has the ability to draw from the sLORETA data and create eight images per second output. As a result, Figures 5-7 contain 22 sequenced images drawn from exposure to the first 2.8-s slide. After analyzing hundreds of stimuli output, we find that in general these 22 images can be further broken down into three separate mental processing zones. While at present the authors are unaware of any supporting literature for these separations, we find that in general the first six or seven set of 0.125 -s images depict the time required for the person to read and comprehend the stimuli. This zone segment varies based on the length of the trigger statement, key words within the stimulus, and individual participant uniqueness. During this zone, we often see initial emotional gamma asymmetry within the frontal lobes. 


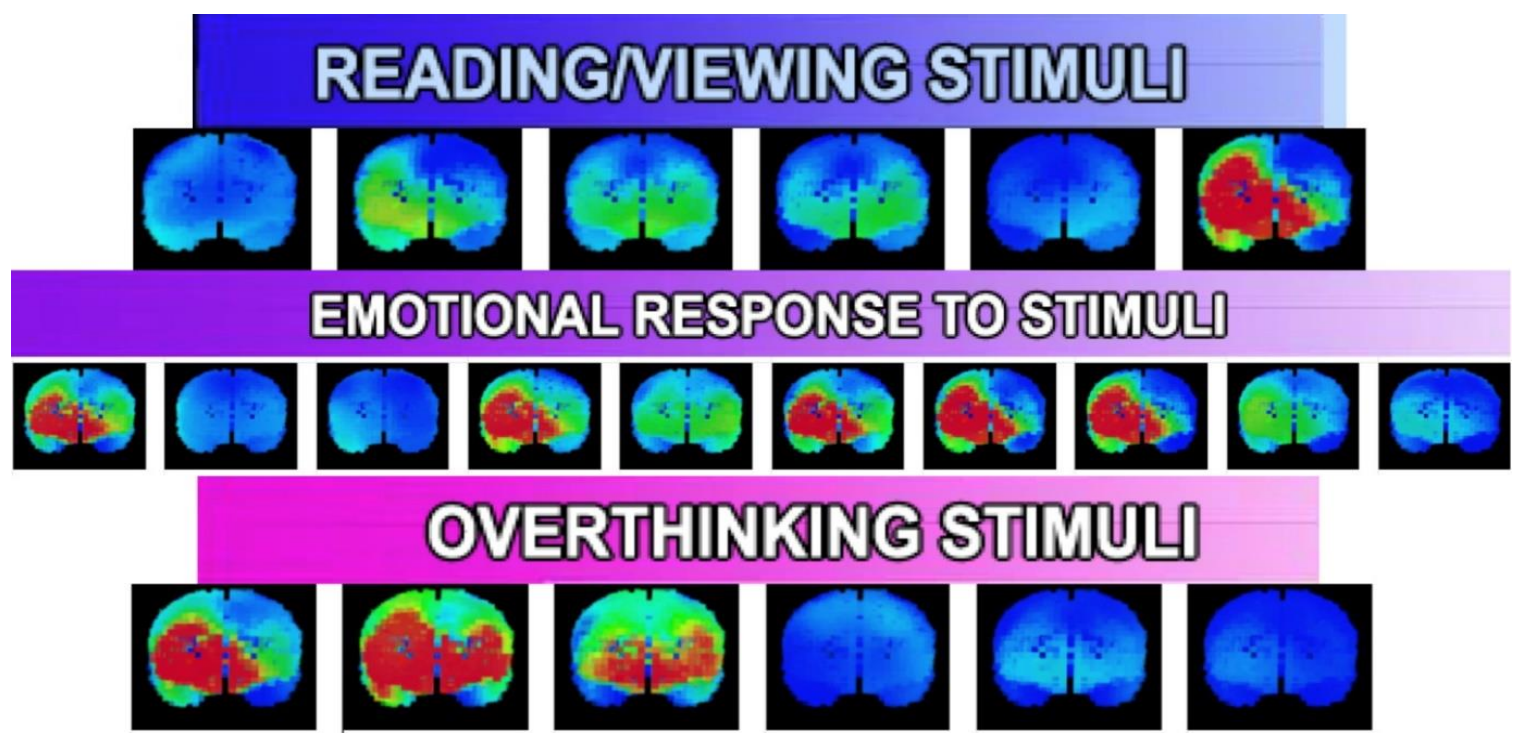

Figure 5a. Brain Disagreement with Entered Statement Response, Example 1.

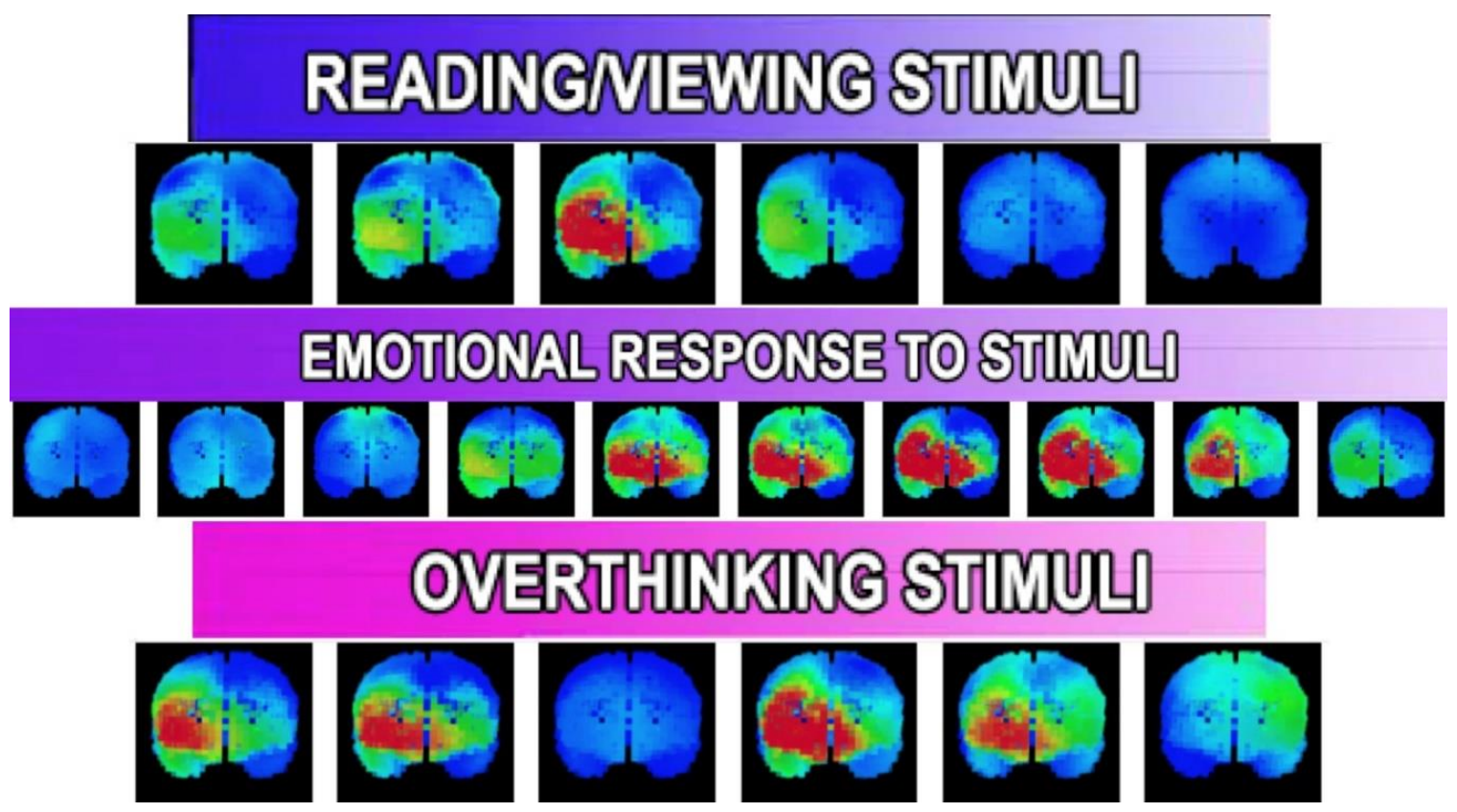

Figure 5b. Brain Disagreement with Entered Statement Response, Example 2.

Both examples in Figure 5 depict a mismatch between the brain reaction and the final marked answer by the participant. Notice the sixth image in Example 1 and the third image in Number 2. Both are right gamma flares that indicate an avoidance, and yet their actual answer was that the statement was very accurate. This right dominance continues throughout all three zones of processing. We are not suggesting that the person is not being honest, but when examining the actual item statement and cross-matching with exploratory factor analysis, both of these items may be exposing a socially acceptable response over a personally descriptive reply. In other words, the item may need to be improved to better align with the intended item purpose. 


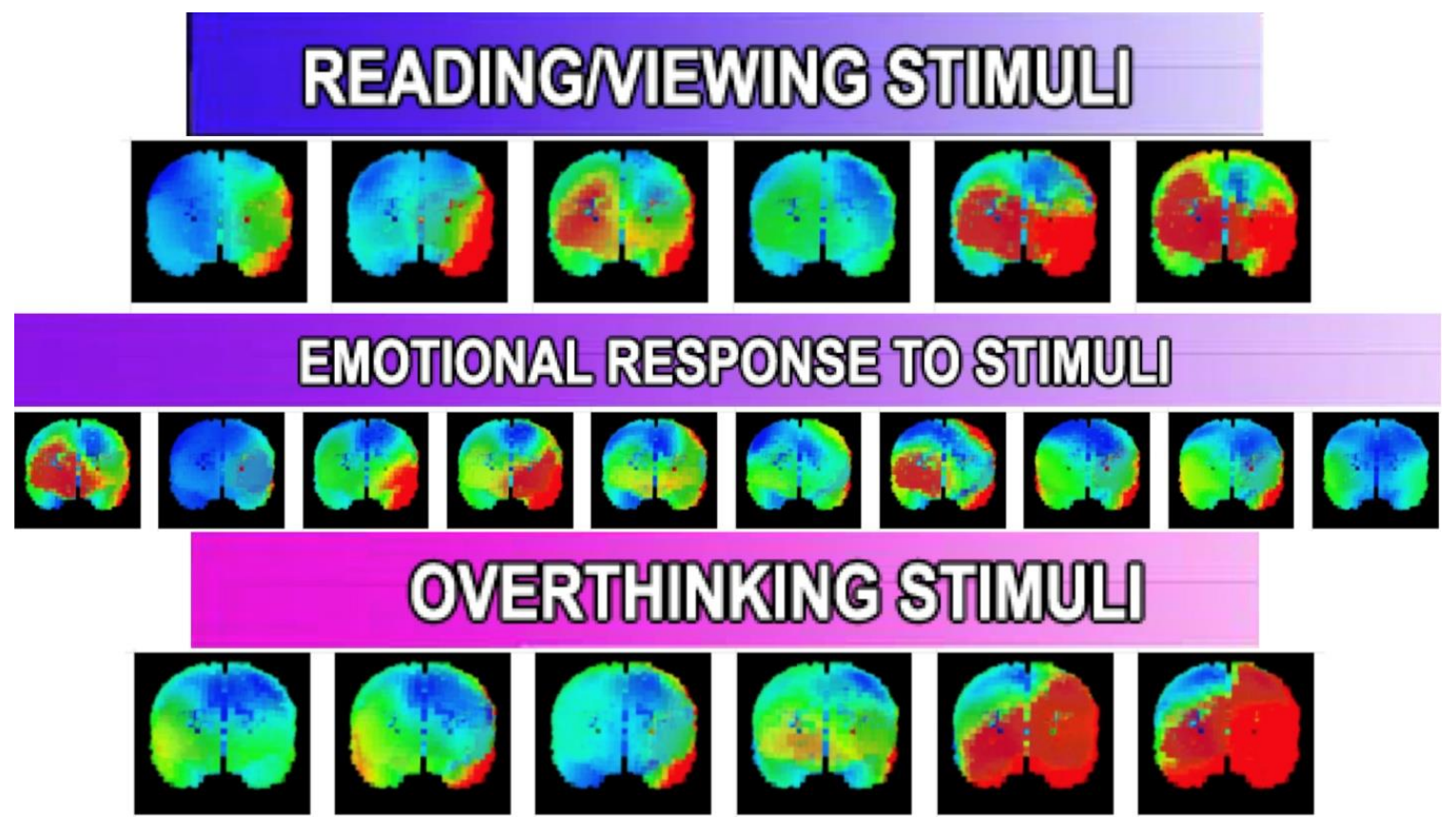

Figure 6a. Confusing Statements, Example 1.

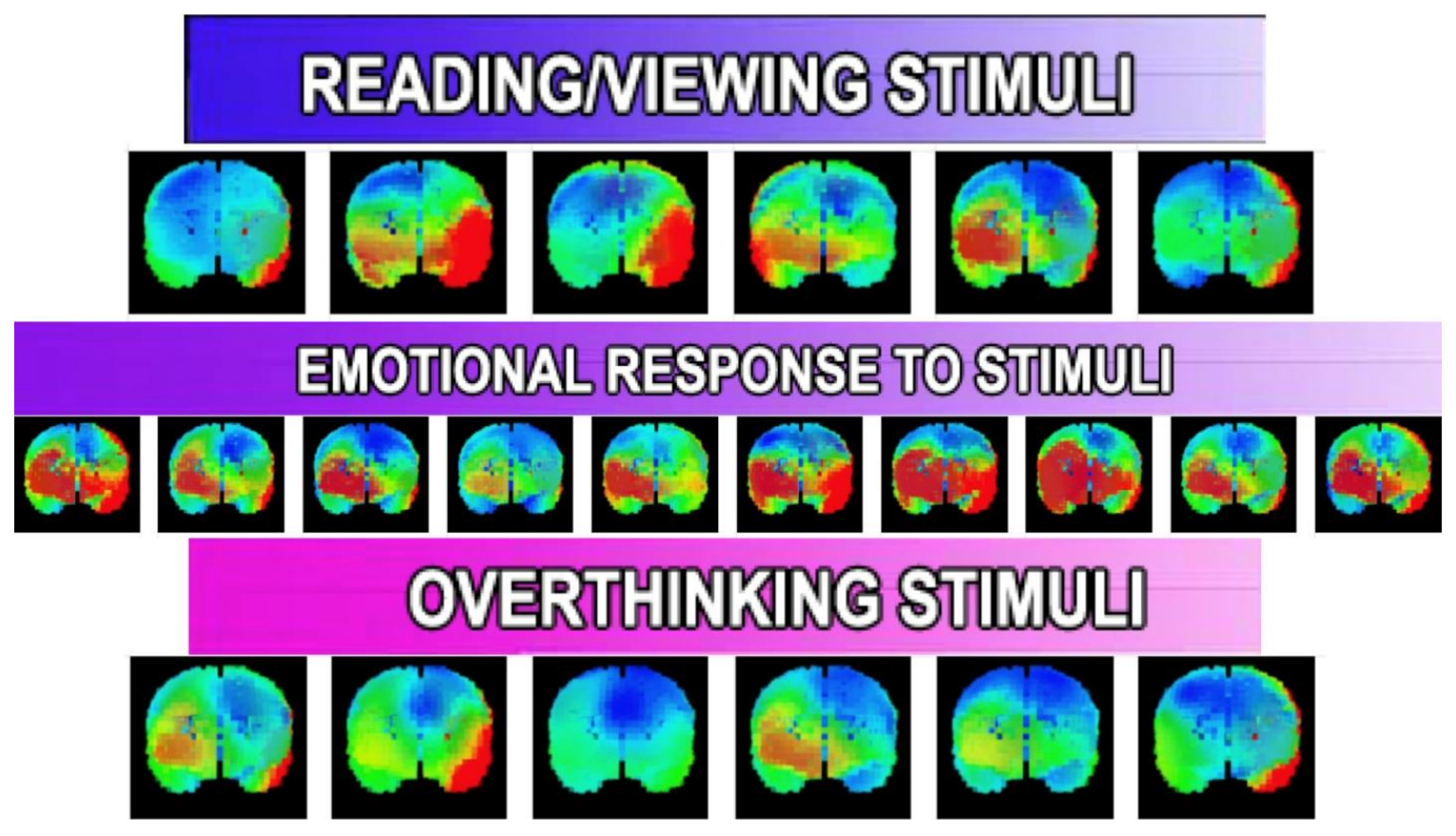

Figure 6b. Confusing Statements, Example 2.

In Figure 6 examples, we see very different brain reactions. Both of these sets of images represent a response to negatively stated stimuli, better known as a reversed item or a double negative. While we will not debate the pros and cons of statements that attempt to address what you are NOT to get at what you are, it is important to recognize that the brain processes this request very differently and, as a result, the item may need further consideration. 


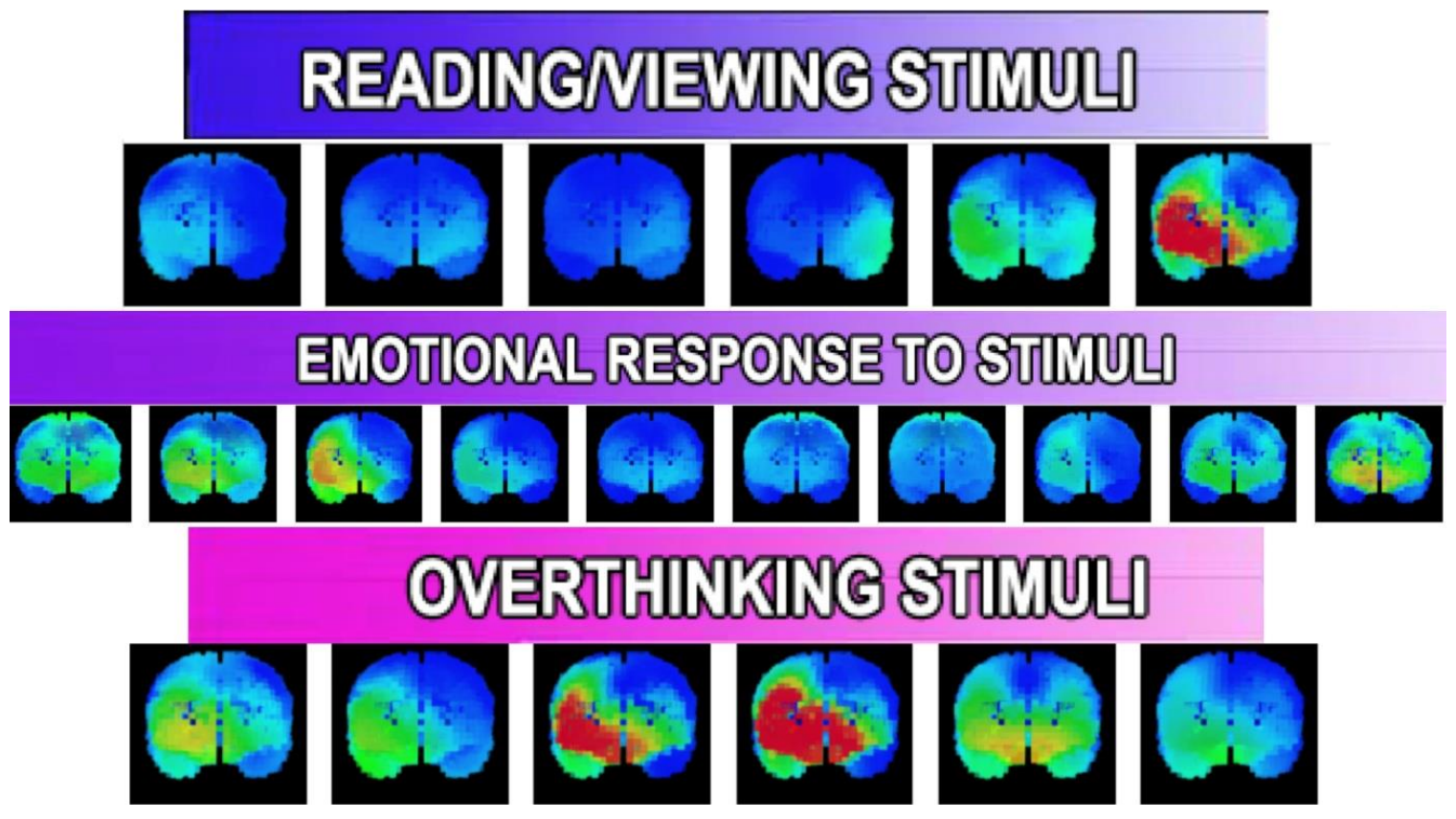

Figure 7a. Neutral Gamma Response, Example 1.

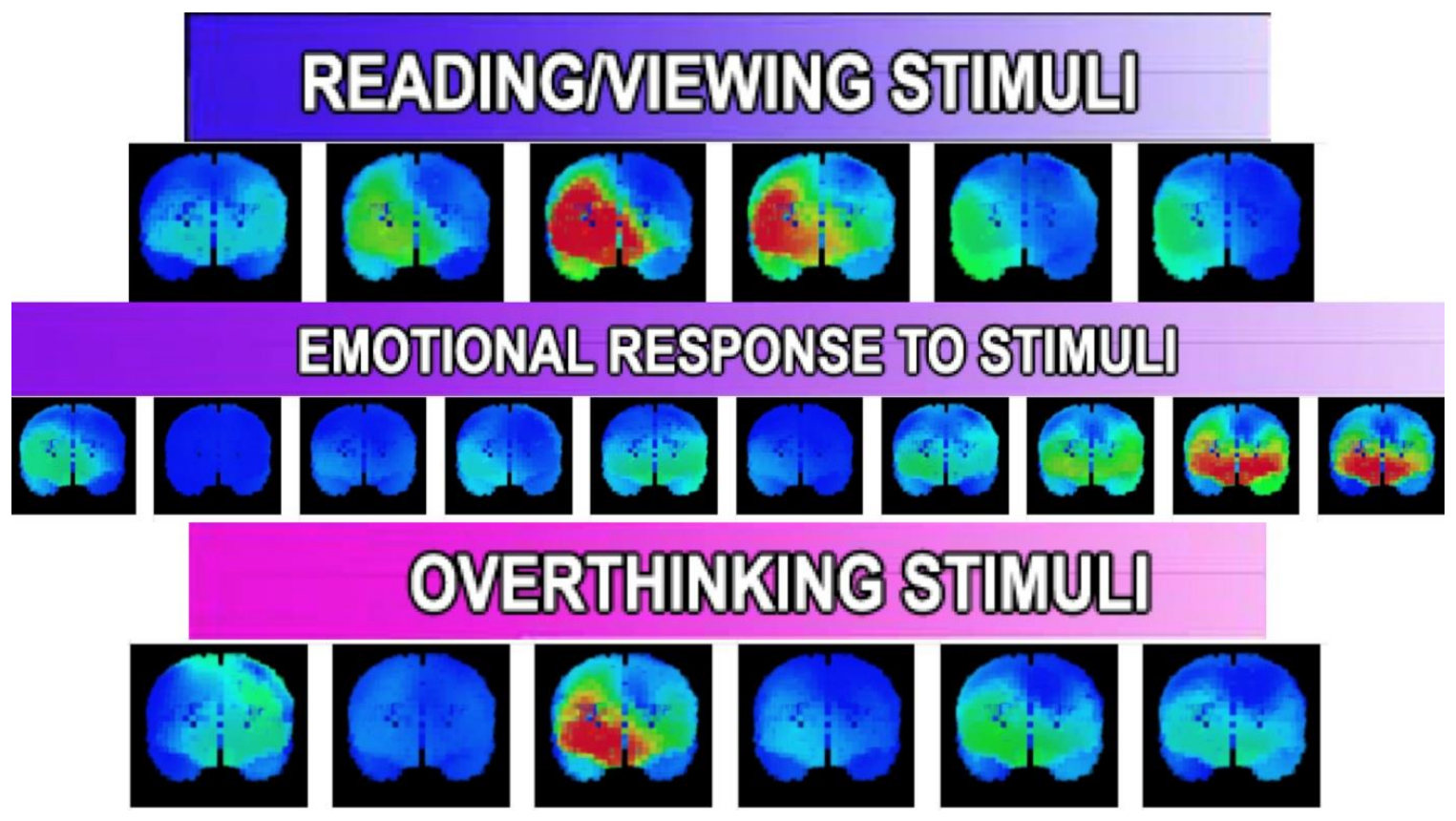

Figure 7b. Neutral Gamma Response, Example 2.

Figure 7 exposes the brain response to an item that was rated neutral on the survey. Notice the lack of gamma response which may indicate a lack of emotional connection to the participant. While a neutral survey may be an accurate answer for an individual, it may also be an indicator of an item with low discrimination. Sets of images such as these in
Figure 7 are flagged for further examination to check for either low factor loading or poor item analysis.

\section{Conclusion}

While response process validity continues to be a challenging assessment design hurdle, this process 
of using sLORETA imaging from EEG data with qEEG analysis and matching these insights to the population assessment analytics are opening new assessment item discussions that both affirm many statistical findings and offer new intriguing explanations regarding respondent mental processing.

At present, this protocol has yielded several insights as we compare item response to actual brain activity, including:

1. Confirmation between survey response and neurological processing

2. Items that may have socially acceptable or "correct" answers and that therefore fail to match brain-processing imagery

3. Mixed brain response to confusing and reverse or double negative assessment items, and

4. Reduced activation for a set of assessment items that many times also exhibit low statistical discrimination.

We are entering a new era where industrial and organizational assessments can be revisited and refined to go beyond the simple exposing of symptoms to bringing to light the causes behind the assessment answers. The day may come when we are able to help people deal with implicit memories, differentiate personal beliefs from socially acceptable responses, and have meaningful conversations about responses that either do or don't match their assessment answers. In the meantime, this additional tool is helping to build and refine assessment tools in ways never before possible.

\section{Author Note}

The authors of the paper are employed by Target Training International Success Insights.

\section{References}

Bonnstetter, B. J., Bonnstetter, R. J., Hebets, D., \& Collura, T. F. (2015). U.S. Patent No. 9,060,702. Validation process for ipsative assessments. Washington, DC: U.S. Patent and Trademark Office.

Bonnstetter, R. J., Hebets, D., \& Wigton, N. L. (2015). Frontal gamma asymmetry in response to soft skills stimuli: A pilot study. NeuroRegulation, 2(2), 70-85, http://dx.doi.org /10.15540/nr.2.2.70

Collura, T. F., Wigton, N. L., Zalaquett, C., Chatters-Smith, S., \& Bonnstetter, R. J. (2016). The value of EEG-based electromagnetic tomographic analysis in human performance and mental health. Biofeedback, 44(2), 58-65. http://dx.doi.org/10.5298/1081-5937-44.2.03

Collura, T. F., Zalaquett, C., \& Bonnstetter, R. J. (2014). Seeing inside the client's mind. Counseling Today, 57(6), 24-27.

Collura, T. F., Zalaquett, C., Bonnstetter, R. J., \& Chatters S. (2014). Towards an operational model of decision-making, emotional regulation, and mental health impact. Advances in Mind-Body Medicine, 28(4), 18-33.

Owen, W. J., Borowsky, R., \& Sarty, G. E. (2004). FMRI of two measures of phonological processing in visual word recognition: Ecological validity matters. Brain and Language, 90(1-3), 40-46. $\quad$ http://dx.doi.org/10.1016/S0093934X(03)00418-8

Sireci, S. G. \& Sukin, T. (2013). Test Validity. In K. F. Geisinger, B. A. Bracken, J. F. Carlson, J.-I. C. Hansen, N. R. Kuncel, S. P. Reise, \& M. C. Rodriguez (Eds.), APA Handbook of Testing and Assessment in Psychology, Volume 1: Test Theory and Testing and Assessment in Industrial and Organizational Psychology (Ch. 4, pp. 61-84). Washington, DC: American Psychological Association. http://dx.doi.org /10.1037/14047-004

TTI Success Insights. (2018, August 21). Style Insights (Behaviors) Instrument Introduction. Retrieved from https://www.ttisuccessinsights.com/products/

Received: August 25, 2018

Accepted: August 31, 2018

Published: September 29, 2018 